ILC

- The Law of Non-Navigational Uses of International Watercourses

INCD

- Desertification Convention
South Africa/EU

335 - Extracts from the Berlin Declaration

366 World Bank

- The World Bank Inspection Panel

\title{
AUTHOR INDEX OF VOLUME 24
}

Abate, Dejen

Abeyratne, R.I.R.

Akhtar, Shahid

Diaz, Ayesha

Gerits, Rob

Gwilliam, H.

Hinssen, Jules

Lang, Wilfried

Lugten, G.
71 Mann-Borghese. Elisabeth

19

235 Miller, Christoph E.

Miller, David

263

71

157

323

254

323

235

254

Oberthür, Sebastian

Peccolo, Gianpaolo

Rau, Johannes

Rest, Alfred

Rummel-Bulska, Iwona

Simoncini, Andrea
56

299

30

70

173,314

13

95 